\title{
A biomimetic Schlemm's canal inner wall: a model to study outflow physiology, glaucoma pathology and high-throughput drug screening
}

Cula N. Dautriche ${ }^{a}$, Dennis Szymanski ${ }^{a}$, Matthew Kerr ${ }^{a}$, Karen Y. Torrejon ${ }^{a}$, Magnus Bergkvist $^{a}$, Yubing Xie ${ }^{a}$, John Danias ${ }^{b}$, W.D. Stamer ${ }^{c, d}$, and Susan T. Sharfstein ${ }^{a, 1}$

${ }^{a}$ Colleges of Nanoscale Science and Engineering, SUNY Polytechnic Institute, 257 Fuller Road, Albany, NY 12203, USA

${ }^{b}$ Departments of Ophthalmology and Cell Biology, SUNY Downstate Medical Center, Brooklyn, New York, 11203, USA

${ }^{c}$ Department of Ophthalmology, Duke University, Durham, NC, 27710, USA

${ }^{d}$ Department of Biomedical Engineering, Duke University, Durham, NC, 27710, USA

${ }^{1}$ To whom all correspondence should be addressed

Susan Sharfstein

Associate Professor of Nanobioscience

SUNY Polytechnic Institute, Colleges of Nanoscale Science and Engineering

257 Fuller Road

Albany, NY 12203

$518-437-8820$

ssharfstein@sunycnse.com 


\section{Abstract}

Glaucoma is a disease that damages the optic nerve, frequently leading to blindness. Elevated intraocular pressure (IOP) is the only modifiable risk factor for glaucoma, which is expected to affect 80 million people by 2020, causing bilateral blindness in over 10 million individuals. Because pathological changes to Schlemm's canal (SC) may account for significant resistance to outflow, there is considerable interest in characterizing and evaluating the Schlemm's canal as a target for glaucoma therapeutics. In conventional, two-dimensional culture, human Schlemm's canal (HSC) cells lose spatial, mechanical and biochemical cues, resulting in altered gene expression and cell signaling than observed in vivo, compromising the clinical relevance of data obtained from such systems. Here, we report, for the first time, that 3D culture of HSC cells on microfabricated scaffolds with defined physical and biochemical cues, rescued expression of key HSC markers, VE-cadherin and PECAM1, and mediated pore formation, crucial for the Schlemm's canal regulation of IOP. We demonstrated that following treatment with the glaucopathogenic agent, TGF- $\beta 2$, HSC cells undergo an endothelial-mesenchymal transition, which together with the increase in extracellular matrix (ECM) proteins might account for the decrease in outflow facility observed in patients with high TGF- $\beta 2$ levels in their aqueous humor. We also demonstrated that unlike 2D cultures, 3D cultures of HSC cells are amenable to gene transfer. Thus, our data imply that 3D culture of HSC cells may be used as a platform to advance our understanding of HSC physiology and pathology and as a model for high-throughput drug and gene screening.

Keywords: Schlemm's canal, glaucoma, tissue engineering, biocompatibility, co-culture, free form fabrication, gene transfer

Running Title: Bioengineering the Schlemm's canal 


\section{Introduction}

Among ocular pathologies, glaucoma remains the second leading cause of blindness and is expected to affect 80 million people worldwide by 2020 , causing bilateral blindness in over 10 million individuals. The primary site of outflow resistance leading to elevated intraocular pressure (IOP) is the juxtacanalicular trabecular meshwork (JCT) and the Schlemm's canal (SC) inner wall. In glaucoma, drainage of aqueous humor into the Schlemm's canal is hindered, leading to an increase in intraocular pressure (IOP) and subsequent damage to the optic nerve, resulting in progressive loss of sight. Several studies have documented pathological changes to the SC inner wall in glaucoma ${ }^{1-4}$, implicating it as a significant contributor to increased outflow resistance ${ }^{5}$ and thus, to elevated IOP. As a result, characterizing the physiology and pathology of the SC inner wall has attracted significant attention and consideration as a possible target for conventional outflow tract-targeted therapies, leading to glaucoma treatment ${ }^{6}$. The SC inner wall is a unique endothelial tissue that is specialized to handle basal to apical transendothelial pressure-dependent flow ${ }^{5}$. Together with the JCT of the human trabecular meshwork (HTM), the human SC (HSC) regulates and maintains proper IOP homeostasis. Much about the SC's physiology and pathology remains poorly understood. This is, in part, due to the lack of an in vitro model to study the SC inner wall and HSC cell biology, physiology, and pathology. Conventional, two-dimensional (2D) cultures of HSC cells on tissue culture plastic are routinely used as laboratory models for understanding HSC cell biology and physiology 7,8 . In such cultures, however, the SC inner wall's micro-architecture, spatial, mechanical, and biochemical cues are lost, resulting in gene expression and cell signaling ${ }^{9}$ that are often different from those observed in vivo. These shortcomings compromise the clinical relevance of data obtained from such systems and speak to the need to generate a model that more closely reproduces the environment of the target tissue for obtaining relevant biomedical data ${ }^{10}$. Here, we report the engineering of a biomimetic 3D SC inner wall that recapitulates the morphological, biochemical and functional characteristics of the HSC, as a model for studying its biology, physiology, and pathology as well as glaucoma drug development. 


\section{Materials and Methods}

Microfabrication of $12 \mu \mathrm{m}$ porous SU-8 scaffolds

Omincoat (MicroChem, Newton, MA), the release layer, was applied onto a clean silicon wafer using a spin coater (Brewer Science, Rolla, MO) at 3,000 rpm for $30 \mathrm{~s}$, and baked on a hot plate at $150{ }^{\circ} \mathrm{C}$ for $5 \mathrm{~min}$. Subsequently, SU-8 2010 (MicroChem, Newton, MA) was applied on top of the release layer by spin-coating to a final thickness of approximately $7 \mu \mathrm{m}$, then baked at $95^{\circ} \mathrm{C}$ for $10 \mathrm{~min}$, and cooled to room temperature. The SU-8 substrate was then exposed through a mask containing the desired patterns using a $150 \mathrm{~mJ} / \mathrm{cm}^{2}$ dose on an EVG 640 I-line Contact Aligner (EV Group, Albany, NY). Finally, the substrate was baked at $95^{\circ} \mathrm{C}$ for $10 \mathrm{~min}$, cooled to room temperature, and placed in SU-8 developer (MicroChem, Newton, MA) for 20 min. The scaffolds were then released following immersion of the substrate into 300 AZ MIF Developer (AZ Electronic Materials USA Corp., Branchburg, NJ). The released, porous SU-8 scaffolds were washed in acetone (Transgene Company, Danvers, MA), and sterilized by soaking in $70 \%$ ethanol for $30 \mathrm{~min}$.

Extracel coating of $12 \mu \mathrm{m}$ porous SU-8 scaffolds

Hystem-C hydrogel kit (ESI BIO, Alameda, CA) contains Glycosil (thiol-modified sodium hyaluronate), Gelin S (thiol-modified gelatin) and Extralink (polyethelyne glycol diacrylate). Glycosil, Gelin $S$ and Extralink solutions were prepared as per manufacturer's instructions. To form the hydrogel, equal volumes of Glycosil and Gelin $S$ were mixed, followed by addition of the Extralink in a 1:4 (Extralink to Gelin S+ Glycosil) volume ratio. Sterilized SU-8 scaffolds were then dipped into the hydrogel solution, removed and allow to gel at room temperature for $15 \mathrm{~min}$.

Cell culture

Primary HSC cells were prepared as previously described ${ }^{7}$. Primary HTM cell cultures from three different donors were studied. The HSC cells were cultured in Dulbecco's modified Eagle's medium (DMEM; Life Technologies, Carlsbad, CA) containing 10\% Premium Select Fetal bovine serum (FBS; Atlanta Biologicals, Lawrenceville, GA), supplemented with penicillin (100 units $/ \mathrm{mL})$, streptomycin $(0.1 \mathrm{mg} / \mathrm{mL})$, and L-glutamine 
(0.292 mg/mL; Life Technologies, Carlsbad, CA). All experiments were conducted using confluent cultures between third and fifth passages.

HSC or HTM cell culture on the SU-8 Scaffolds

For establishment of the monolayer formation, $5 \times 10^{4}$ primary HSC cells were seeded on Extracel-coated $12 \mu \mathrm{m}$-pore SU-8 scaffolds and cultured for 10 days (HSC) or 14 days (HTM) to confluence. Upon reaching confluence, HSC cells were fixed for immunohistochemistry $(\mathrm{IHC})$ analysis or SEM or used for drug treatment and/or perfusion studies.

F-actin staining, immunocytochemistry and microscopy

The engineered HSC cell monolayer was washed with 1x phosphate buffered saline (PBS) three times and fixed in 4\% paraformaldehyde/PBS for 15 min. After which, the construct was then washed three times in 1X PBS and permeabilized in 1\% Triton X-100 in 1x PBS and blocked with serum buffer containing 5\% FBS in PBS buffer for 1 hour. The transfected cells were then incubated overnight at $4^{\circ} \mathrm{C}$ with phalloidin for $\mathrm{F}$-actin staining and/or the respective primary antibodies (Table 1).

The transfected cells were then washed five times in 1x PBS and followed by incubation with appropriate Alexa fluor-conjugated secondary antibodies in 1x PBS at room temperature for 1 hour. Secondary antibodies included goat anti-mouse Alexa Fluor (GAM) 594 (1/200), goat anti-rabbit Alexa Fluor (GAR) 488 (1/100), goat anti-rabbit Alexa Fluor (GAR) 594 (1/200), donkey anti-goat Alexa Fluor (DAG) 594 (1/200) (Life Technologies, Carlsbad, CA). Finally, the monolayer was washed three times with $1 \mathrm{x}$ PBS, mounted onto glass slides with Prolong Gold Antifade Reagent (Life Technologies, Carlsbad, CA). Laser scanning confocal microscopy was performed using a Leica SP5 confocal microscope (Leica Microsystems, Mannheim, Germany), and images were acquired at 40X magnification with an oil-immersion objective. Confocal images were processed using Imaris software 7.7 (Bitplane Inc, South Windsor, CT), and all images within a given experiment were captured using the same laser intensity and gain settings in order to be able to compare intensities across samples. 
Scanning electron microscopy

Surface analysis of the confluent HSC cell monolayer construct was conducted using a LEO 1550 field emission scanning electron microscope (SEM) (Leo Electron Microscopy Ltd., Cambridge, UK). Constructs were fixed for $2 \mathrm{~h}$ at room temperature in a $3 \%$ glutaraldehyde solution in $0.1 \mathrm{M}$ phosphate buffer containing $0.1 \mathrm{M}$ sucrose. Constructs were then washed three times in $0.1 \mathrm{M}$ phosphate buffer, and were chemically dehydrated in a graded ethanol series and slowly infiltrated with a graded hexamethyldisilazane (HMDS)-ethanol series (25\%, 50\%, 75\%, and 100\% HMDS) for drying. Finally, dehydrated constructs were mounted on $1-\mathrm{cm}^{2}$ stubs using carbon tape and sputter-coated with $5 \mathrm{~nm}$ gold-palladium to avoid charging the sample. Images were acquired at a working distance of $3 \mathrm{~mm}$ with an acceleration gun voltage of $3 \mathrm{keV}$.

Quantitative real-rime PCR (qRT-PCR) analysis

PCR probes and primers (Table 2) were generated using Integrated DNA Technologies probe/primer design software (http://www.idtdna.com/pages/products/geneexpression/ primetime-qpcr-assays-and-primers).

Total RNA was extracted from confluent HSC cultured on tissue culture plastic or Extracel-coated tissue culture plastic plates or Extracel-coated SU-8 scaffolds cells using RNeasy MiNi Kit (50) as per the manufacturer's instructions (Qiagen, Hilden, Germany). RNA was quantified using a NanoDrop ND1000 Spectrophotometer (Thermo Scientific, Wilmington, DE). qRT-PCR was carried out as directed using TaqMan RNAto- $\mathrm{C}_{\mathrm{T}}{ }^{\mathrm{TM}}$ 1-Step Kit (Applied Biosystems, Carlsbad, CA) using $100 \mathrm{ng}$ of RNA for each samples. qRT-PCR was performed on an AB StepOnePlus Real Time PCR system (Life Technologies, Carlsbad, CA) using an RT step (48 ${ }^{\circ} \mathrm{C}$ for $15 \mathrm{~min}$ ) followed by an enzyme activation step $\left(95^{\circ} \mathrm{C}\right.$ of $\left.10 \mathrm{~min}\right)$, then 40 cycles of $15 \mathrm{~s}$ denaturation at $95^{\circ}$ and $1 \mathrm{~min}$ anneal/extend at $60^{\circ} \mathrm{C}$. Relative quantitation data analysis was performed using the comparative quantification method, $\triangle \Delta \mathrm{Ct}$, with $\mathrm{GAPDH}$ as the endogenous reference. Experiments were run in triplicate and the average values are presented as mean \pm SEM, with $P$ values $<0.001$ considered significant. 


\section{Perfusion Studies}

At day 14, the HSC cell monolayer constructs were secured in the perfusion chamber and perfused at varying flow rates $(2,4,8,10,14,20 \mu \mathrm{L} / \mathrm{min})$ in the apical-to-basal direction for $6 \mathrm{~h}$, with perfusion medium consisting of DMEM supplemented with penicillin (100 units $/ \mathrm{mL})$, streptomycin $(0.1 \mathrm{mg} / \mathrm{mL})$, and L-glutamine $(0.292 \mathrm{mg} / \mathrm{mL}$; Life Technologies, Carlsbad, CA). The temperature of the chamber was maintained at $34^{\circ} \mathrm{C}$. Back pressure was continuously monitored with a pressure transducer and recorded. For the treatment with TGF- $\beta 2$ (R\&D systems), samples were pretreated with $2.5 \mathrm{ng} / \mathrm{ml}$ of TGF- $\beta 2$ for $48 \mathrm{hrs}$ and then were perfused with medium supplemented with $2.5 \mathrm{ng} / \mathrm{ml}$ of TGF- $\beta 2$ in DMEM at varying flow rates $(\mu \mathrm{L} / \mathrm{min})$ in the apical-to-basal direction for 6 h.

\section{Statistical Analysis}

The average values are presented as mean \pm SEM, with $P$ values $<0.001$ considered significant. To show significant mRNA expression changes compared to untreated controls, one-way ANOVA was used (GraphPad Prism 6.02; GraphPad Software, Inc., La Jolla, CA).

\section{Results and Discussion}

The SC inner wall lies directly against the JCT, resting on an incomplete basal lamina with considerable areas of the basal membrane not supported by ECM, but rather in direct contact with the open spaces of the JCT and aqueous humor flow ${ }^{11}$. Attempting to recreate this situation in vitro, we evaluated HSC cell growth and development on gelatin-coated, highly porous, micro-patterned SU-8 scaffolds ${ }^{12}$ with pore sizes of 7, 12 or $15 \mu \mathrm{m}$. Scanning electron microscopy (SEM) analysis showed that few HSC cells attached and grew on the gelatin-coated SU-8 scaffold even after 10 days in culture, irrespective of pore size (Fig. 1a, left panels). Given the intricate role of the extracellular matrix $(E C M)$ in providing biochemical cues for cell growth and development, we hypothesized that hydrogels of ECM components would provide the necessary biochemical cues for HSC cell growth and development. Hyaluronic acid (HA) was tested due to its abundance in the JCT region of the conventional outflow tract 
13. More importantly, the loss of $H A$ is associated with aging and glaucoma as $H A$ is depleted in all layers of all regions of the primary open-angle glaucoma JCT ${ }^{14}$. These findings suggest that HA or HA signaling may play an important role in providing the proper biochemical cues suitable for HSC cell growth, development and function. The HA-based hydrogel, Extracel (HA-Gelin S) (Fig. 1b) was selected to coat our porous SU-8 scaffolds (Fig. 1b) to determine whether it would facilitate the growth and maintain the differentiation of HSC cells. As demonstrated by SEM analysis in Fig. 1a (right panels), Extracel hydrogel coating of the microporous scaffolds supported the growth of HSC cells into a confluent monolayer after 10 days in culture, irrespective of scaffold pore size. We selected the $12 \mu \mathrm{m}$-pore SU-8 scaffolds for the remainder of these studies, since they better supported HTM cell growth in our previous studies ${ }^{12}$. After 14 days in culture, HSC cells on Extracel-coated SU-8 (ESU-8) scaffolds established a continuous, confluent monolayer, consistent with that of the inner wall of the SC (Fig. 1c). In addition, live cell counts at day 7 of culture demonstrated that HSC cells on ESU8 scaffolds exhibited a 2 -fold increase in proliferation as compared to the gelatin-coated SU-8 scaffolds, consistent with the SEM observation (Fig. 1d), highlighting the importance of selecting the correct ECM materials for in vitro culture systems.

We next characterized the engineered SC inner wall by studying cytoskeletal architecture and the expression of key HSC cell markers, including vascular endothelial cadherin (VE-cadherin), CD31, and fibulin- $2^{8},{ }^{15}$. Cytoskeletal visualization via F-actin staining revealed a greater degree of fiber alignment in HSC cells cultured on our ESU8 system compared to those cultured on Extracel-coated glass coverslips, while HSC cells cultured on gelatin-coated glass coverslips did not exhibit cytoskeletal alignment (Fig. 2a). Greater fiber alignment is indicative of a more HSC tissue-like behavior ${ }^{16}$, facilitating better monolayer organization and cell-cell adhesion for its blood-aqueous barrier function ${ }^{17}$. HSC cells are known to lose characteristic expression of VEcadherin and CD31 in conventional 2D tissue culture ${ }^{8}$. Immunocytochemistry analysis of the endothelial cell-junction marker, CD31 (Fig. 2a) and VE-cadherin (Fig. 2a) demonstrated that HSC cells on ESU-8 scaffolds regained characteristic expression and displayed proper localization of these two key HSC cell characteristic markers. These results suggest that ESU-8 scaffolds provide both the necessary biochemical and 
spatial cues for proper HSC cell morphology and function, consistent with the in vivo phenotype. We also analyzed the expression of the secreted ECM glycoprotein, fibulin2, which revealed a more grainy pattern on ESU-8 scaffolds, possibly due to increased secretion and retention in the pores of the ESU-8 scaffold (Fig. 2a). Increased gene expression of the HSC cell characteristic markers was further confirmed via qRT-PCR, demonstrating dramatic upregulation of fibulin-2, CD31, and VE-cadherin when compared to cells cultured on tissue culture plastic in conventional 2D cultures (Fig. 2b).

Given the SC inner wall's continuous exposure to stress induced by trans-endothelial flow of aqueous humor, we next investigated the effect of basal-apical flow on this engineered SC layer, mimicking the in vivo outflow physiology. The resulting hydraulic conductivity of the engineered SC inner wall was calculated to be $0.046 \mu \mathrm{l} / \mathrm{min} / \mathrm{mm} \mathrm{Hg} /$ $\mathrm{mm}^{2}$, which is roughly $1 / 3$ of the previously described biomimetic JCT layer of the HTM 12. The HTM layer exhibits an outflow facility of $0.104 \mu \mathrm{l} / \mathrm{min} / \mathrm{mm} \mathrm{Hg} / \mathrm{mm}^{2}$, roughly approximating the $0.35 \mu \mathrm{l} / \mathrm{min} / \mathrm{mm} \mathrm{Hg} / \mathrm{mm}^{2}$ of the in vivo value, while the HSC monolayer outflow is $0.046 \mu \mathrm{l} / \mathrm{min} / \mathrm{mm} \mathrm{Hg} / \mathrm{mm}^{2}$. This resistance to flow confirms the integrity of the endothelial layer resulting from expression of its tight junction proteins, CD31 and VE-cadherin (Fig. 2c). A hallmark of HSC cells, which differentiate them from other endothelial cells, is their ability to form shear stress-induced paracellular and transcellular vacuoles to mediate aqueous humor homeostasis ${ }^{18}$. SEM analysis confirmed the formation of micron pores following perfusion at $4 \mu \mathrm{l} / \mathrm{min}$ for $6 \mathrm{~h}$ (Fig. 2d). In addition, there was an increase in pore formation in the engineered SC inner layer with increasing flow rate during perfusion (data not shown), which is consistent with increasing in biomechanical stress translating into greater pore density ${ }^{18}$.

Having demonstrated that the microporous scaffold-hydrogel culture system facilitated in vivo-like HSC physiology, we further determined whether the culture system is affected by pharmacologic agents that are known to cause IOP elevation. Responses of HTM cells to the cytokine TGF- $\beta 2{ }^{19}$ are among the most studied and well characterized. However, the specific responses of HSC cells in vivo to these agents are not as well characterized. We evaluated the response of the engineered SC layer to 
TGF- $\beta 2$. We assessed TGF- $\beta 2$ characteristic induction of ECM proteins (e.g. fibronectin) and carried out a dose-response study. TGF- $\beta 2$ treatment resulted in increased actin stress-fiber formation (Fig. 3a) and the induction of fibronectin expression (Fig. 3b, e). More notably, TGF- $\beta 2$ induced an endothelial/mesenchymal transition (EndMT) ${ }^{20}$ of the HSC cells as demonstrated by down-regulation of the endothelial cell marker, VE-cadherin (Fig. 3c), expression of the mesenchymal marker, a-SMA (Fig. 3c, d) and ECM protein expression (Collagens I and IV) (Fig. 3c,e). Together, these data support the feasibility of using an engineered SC layer to study the response of HSC cells to pathogenic or therapeutic agents.

With the advent of siRNA biomolecules as a possible glaucoma treatment ${ }^{21}$, we explored whether the engineered HSC layer is susceptible to gene silencing. To assess the efficiency of siRNA uptake the engineered SC layer was transfected with FITC-siRNA. Nearly $100 \%$ of the cells incorporated FITC-siRNA 24 hrs post-transfection (Fig. 4a). This is surprising as HSC cells in 2D cultures are difficult to transfect, achieving roughly $1 \%$ transfection efficiency (Fig. S2d). The observed efficiency could be explained by apical and basal access to media in this culture system as well as the maintenance of a more in vivo-like physiology. To confirm siRNA down-regulation, we assessed $\beta$-actin expression 48 hrs post transfection with $200 \mathrm{nM} \beta$-actin siRNA (Fig. 4b). The observed $\beta$-actin downregulation suggests that si-RNA can be used for gene silencing in the SC inner wall endothelium.

\section{Conclusions}

We have successfully engineered and characterized a biomimetic SC inner wall as a potential platform to advance our understanding of HSC physiology and pathology, and model for high-throughput drug and gene screening. We demonstrated that $3 \mathrm{D}$ culture of HSC cells on our scaffold-hydrogel system retains much of the in vivo morphology, characteristics, and behaviors that these cells normally lose during conventional $2 \mathrm{D}$ culture. Characterization of the cellular responses of the 3D HSC cultures to the glaucopathogenic agent, TGF- $\beta 2$, revealed an endothelial-mesenchymal transition, which, together with the increase in ECM proteins, might account for the decrease in outflow facility observed in patients with high TGF- $\beta 2$ levels in their aqueous humor ${ }^{19,22}$. These 
data demonstrate how spatial and biomechanical cues from micro-patterned substrates as well as extracellular signals can help provide the proper signaling to mediate in vitro biomimetic characteristics and responses of a specific tissue. Since the SC inner wall exists in vivo in close apposition to the JCT layer of the TM, the ability to bioengineer an in vitro model of the SC inner wall will allow us to further recapitulate cell-cell signaling and paracrine signaling through 3D co-culture of HTM and HSC cells on the microfabricated scaffold-hydrogel system in the near future.

\section{Acknowledgements}

This work was supported in part by a grant from the SUNY Technology Acceleration Fund, by the National Eye Institute of the National Institutes of Health under award numbers R01EY022359 to WDS and R01EY20670 to JD, and by a Research to Prevent Blindness challenge grant to the Department of Ophthalmology, SUNY Downstate. KYT is the recipient of a National Science Foundation Graduate Fellowship. The authors would like to thank Kristin M Perkumas for isolation and culture of human Schlemm's canal cells. 


\section{References}

1 Allingham, R. R., de Kater, A. W. \& Ethier, C. R. Schlemm's canal and primary open angle glaucoma: correlation between Schlemm's canal dimensions and outflow facility. Exp Eye Res 62, 101-109 (1996).

2 Hann, C. R., Vercnocke, A. J., Bentley, M. D., Jorgensen, S. M. \& Fautsch, M. P. Anatomic Changes in Schlemm's Canal and Collector Channels in Normal and Primary Open-Angle Glaucoma Eyes Using Low and High Perfusion Pressures. Invest Ophthalmol Vis Sci 55, 5834-5841, doi:10.1167/iovs.14-14128 (2014).

3 Allingham, R. R. et al. The relationship between pore density and outflow facility in human eyes. Invest Ophthalmol Vis Sci 33, 1661-1669 (1992).

4 Johnson, M. et al. The pore density in the inner wall endothelium of Schlemm's canal of glaucomatous eyes. Invest Ophthalmol Vis Sci 43, 2950-2955 (2002).

5 Stamer, W. D. et al. Biomechanics of Schlemm's canal endothelium and intraocular pressure reduction. Prog Retin Eye Res, doi:10.1016/j.preteyeres.2014.08.002 (2014).

6 Karpinich, N. O. \& Caron, K. M. Schlemm's canal: more than meets the eye, lymphatics in disguise. J Clin Invest 124, 3701-3703, doi:10.1172/jci77507 (2014).

7 Stamer, W. D., Roberts, B. C., Howell, D. N. \& Epstein, D. L. Isolation, culture, and characterization of endothelial cells from Schlemm's canal. Invest Ophthalmol Vis Sci 39, 18041812 (1998).

8 Perkumas, K. M. \& Stamer, W. D. Protein markers and differentiation in culture for Schlemm's canal endothelial cells. Exp Eye Res 96, 82-87, doi:10.1016/j.exer.2011.12.017 (2012).

9 Zhou, L., Zhang, S. R. \& Yue, B. Y. Adhesion of human trabecular meshwork cells to extracellular matrix proteins. Roles and distribution of integrin receptors. Invest Ophthalmol Vis Sci 37, 104113 (1996).

10 Pampaloni, F., Reynaud, E. G. \& Stelzer, E. H. The third dimension bridges the gap between cell culture and live tissue. Nat Rev Mol Cell Biol 8, 839-845, doi:10.1038/nrm2236 (2007).

11 Tamm, E. R. The trabecular meshwork outflow pathways: structural and functional aspects. Exp Eye Res 88, 648-655, doi:10.1016/j.exer.2009.02.007 (2009).

12 Torrejon, K. Y. et al. Recreating a human trabecular meshwork outflow system on microfabricated porous structures. Biotechnol Bioeng 110, 3205-3218, doi:10.1002/bit.24977 (2013).

13 Keller, K. E. \& Acott, T. S. The Juxtacanalicular Region of Ocular Trabecular Meshwork: A Tissue with a Unique Extracellular Matrix and Specialized Function. J Ocul Biol 1, 3 (2013).

14 Knepper, P. A., Goossens, W. \& Palmberg, P. F. Glycosaminoglycan stratification of the juxtacanalicular tissue in normal and primary open-angle glaucoma. Invest Ophthalmol Vis Sci 37, 2414-2425 (1996).

15 Heimark, R. L., Kaochar, S. \& Stamer, W. D. Human Schlemm's canal cells express the endothelial adherens proteins, VE-cadherin and PECAM-1. Curr Eye Res 25, 299-308 (2002).

16 Ethier, C. R., Read, A. T. \& Chan, D. Biomechanics of Schlemm's canal endothelial cells: influence on F-actin architecture. Biophys J 87, 2828-2837, doi:10.1529/biophysj.103.038133 (2004).

17 Overby, D. R., Stamer, W. D. \& Johnson, M. The changing paradigm of outflow resistance generation: towards synergistic models of the JCT and inner wall endothelium. Exp Eye Res 88, 656-670, doi:10.1016/j.exer.2008.11.033 (2009).

18 Overby, D. R. et al. Altered mechanobiology of Schlemm's canal endothelial cells in glaucoma. Proc Natl Acad Sci U S A 111, 13876-13881, doi:10.1073/pnas.1410602111 (2014).

19 Gottanka, J., Chan, D., Eichhorn, M., Lutjen-Drecoll, E. \& Ethier, C. R. Effects of TGF-beta2 in perfused human eyes. Invest Ophthalmol Vis Sci 45, 153-158 (2004). 
20 van Meeteren, L. A. \& ten Dijke, P. Regulation of endothelial cell plasticity by TGF-beta. Cell Tissue Res 347, 177-186, doi:10.1007/s00441-011-1222-6 (2012).

21 Zhang, K., Zhang, L. \& Weinreb, R. N. Ophthalmic drug discovery: novel targets and mechanisms for retinal diseases and glaucoma. Nat Rev Drug Discov 11, 541-559, doi:10.1038/nrd3745 (2012).

22 Wordinger, R. J. et al. Effects of TGF-beta2, BMP-4, and gremlin in the trabecular meshwork: implications for glaucoma. Invest Ophthalmol Vis Sci 48, 1191-1200, doi:10.1167/iovs.06-0296 (2007). 


\section{Figure Captions}

Figure 1: Evaluation of human Schlemm's canal cell growth on well defined, microfabricated, porous SU-8 scaffolds. (a) Scanning electron microscopy (SEM) images showing that HSC cell growth on SU-8 scaffolds depended on the coating for biochemical signals and was independent of the pore size. Scale bar $=100 \mu \mathrm{m}$. (b) Schematic of the polymerization scheme for Extracel (HA-GelinS). (c) SEM images of SU-8 scaffolds (12- $\mu \mathrm{m}$ pores) coated with $1 \%$ gelatin or Extracel (ESU-8) (top panel). SEM images revealing establishment of a continuous HSC monolayer on the Extracelcoated SU-8 scaffold (bottom panel). Scale bar $=100 \mu \mathrm{m}$. (d) Live cell count demonstrating over 2-fold increase in HSC cell growth on ESU-8 scaffolds compared to gelatin-coated scaffolds. * $(P<0.001$, Extracel vs $1 \%$ gelatin) Mean $\pm S D$ with $N=4$.

Figure 2: Characterization of HSC cell development on ESU-8 (12- $\mathrm{mm}$ pore) scaffolds. (a) F-actin staining, revealing fiber alignment of the HSC cells on the Extracel-coated SU-8 scaffold. HSC cells cultured on the ESU-8 scaffold regained expression of cell characteristic marker, CD31, an expression that is lost in 2D culture on glass coverslips as well as exhibited proper expression and distribution of cell characteristic marker, VE-cadherin, when compared to 2D culture on glass coverslip. HSC cells cultured on the ESU-8 scaffold maintained expression of cell characteristic marker, fibulin 2. Scale bar $=100 \mu \mathrm{m}$. (b) qRT-PCR analysis confirming Extracelinduced upregulation of characteristic HSC markers on ESU-8 scaffolds. ${ }^{*}(P<0.001$, ESU-8 vs $2 \mathrm{D}$ culture), $\#\left(\mathrm{P}<0.001\right.$, ESU-8 vs $2 \mathrm{D}$ culture + Extracel), ${ }^{* *}(\mathrm{P}<0.001,2 \mathrm{D}$ culture + Extracel vs $2 \mathrm{D}$ culture). Mean $\pm \mathrm{SD}$ with $\mathrm{N}=6$. (c) Outflow study demonstrating hydraulic conductivity of the engineered SC inner wall was calculated to be $0.104 \mu \mathrm{l} /$ $\mathrm{min} / \mathrm{mm} \mathrm{Hg} / \mathrm{mm}^{2}$, which is roughly $1 / 3$ of the previously described biomimetic JCT layer of the HTM, an indication of endothelial integrity. (d) SEM images revealing formation of vacuole-like micron-sized pores in the engineered SC inner wall following perfusion at $4 \mu \mathrm{l} / \mathrm{min}$ for $6 \mathrm{~h}$.

Figure 3: Evaluation of the engineered Schlemm's canal inner wall as a model for drug screening. (a, b) Confocal images of engineered SC layer revealing induced fibronectin expression (a) and increased stress fiber formation after treated with 1.4 and $2.5 \mathrm{ng} / \mathrm{ml} \mathrm{TGF-} \beta 2$. (c) Gene expression of the engineered SC layer after treated with $2.5 \mathrm{ng} / \mathrm{ml}$ TGF- $\beta 2$ revealing down-regulated of the endothelial markers, VE-cadherin, CD31, as well as up-regulation of collagen I and the mesenchymal marker, $\alpha$-SMA qRT-PCR and IHC (d). ${ }^{*} P>0.001$, Mean \pm SD with $N=6$. Scale bar $=100 \mu \mathrm{m}$. (e) qRT$\mathrm{PCR}$ analysis confirming up-regulation of fibronectin expression and collagen IV following TGF- $\beta 2$ treatment. * $P>0.001$, Mean $\pm S D$ with $N=6$. (f) Schematic diagram of possible endothelial-mesenchymal transition through TGF- $\beta$ signaling mechanism in the engineered SC inner wall, leading to the characteristic decrease in outflow facility of glaucoma.

Figure 4: Evaluation of the engineered Schlemm's canal inner wall as a model for gene transfer. (a) Gene transfer to the engineered SC layer revealing nearly $100 \%$ transfection efficiency following transfection with $200 \mathrm{nM}$ FITC-siRNA (scale bar $=100$ $\mu \mathrm{m})$ and $\beta$-actin down-regulation $48 \mathrm{~h}$ post transfection with $200 \mathrm{nM}$ si-RNA against $\beta$ actin confirmed by qPCR (b, ${ }^{*} P>0.001$, Mean $\pm S D$ with $N=6$ ). 
Tables

Table 1. Cytochemistry reagents

\begin{tabular}{|l|l|l|l|l|}
\hline \multicolumn{1}{|c|}{ Name } & Species & Dilution & \multicolumn{1}{c|}{ Company } & $\begin{array}{c}\text { Secondary } \\
\text { used }\end{array}$ \\
\hline $\begin{array}{l}\text { Atto-488 conjugated } \\
\text { phalloidin }\end{array}$ & & $1 / 40$ & Sigma Aldrich & \\
\hline a- SMA & Mouse & $1 / 500$ & Abcam, Cambridge MA & GAM 594 \\
\hline Fibronectin & Mouse & $1 / 100$ & Abcam, Cambridge MA & GAM 594 \\
\hline Fibulin 2 & Goat & $1 / 200$ & $\begin{array}{l}\text { Santa Cruz } \\
\text { Biotechnology }\end{array}$ & DAG 594 \\
\hline CD 31 & Rabbit & $1 / 20$ & Abcam, Cambridge MA & GAR 594 \\
\hline VE-cadherin & Rabbit & $1 / 400$ & Cell Signaling & GAR 488 \\
\hline
\end{tabular}

Table 2. qRT-PCR primers

\begin{tabular}{|l|l|l|l|}
\hline $\begin{array}{c}\text { NCBI Gene } \\
\text { Symbol }\end{array}$ & \multicolumn{1}{|c|}{$\begin{array}{c}\text { Reference } \\
\text { Sequence \# }\end{array}$} & \multicolumn{1}{|c|}{ Forward } & \multicolumn{1}{c|}{ Reverse Primer } \\
\hline FN1 & NM_212482 & GTCTTGTGTCCTGATCGTTG & AGGCTGGATGATGGTAGATTG \\
\hline CDH5 & NM_001795(1) & GACAGCGTTCTCACACACT & AGCCAAAGAACTGGATTCC \\
\hline ACTA2 & NM_001613 & CACGAAGCTCATTGTAGAAAGAG & GCACAGAGCAAAAGAGGAATC \\
\hline COL41A & NM_001845 & CCTTTGTGCCATTGCATCC & GAACAAAAGGGACAAGAGGAC \\
\hline PECAM1 & NM_000442(1) & ATTGCTCTGGTCACTTCTCC & CAGGCCCATTGTTCCC \\
\hline COL1A1 & NM_000088 & GGTTGATTTCTATCATAGCCAT & CTGGACAGCCTGGACTTC \\
\hline GAPDH & NM_002046(1) & TGTAGTTGAGGTCAATGAAGGG & ACATCGCTCAGACACCATG \\
\hline
\end{tabular}


․

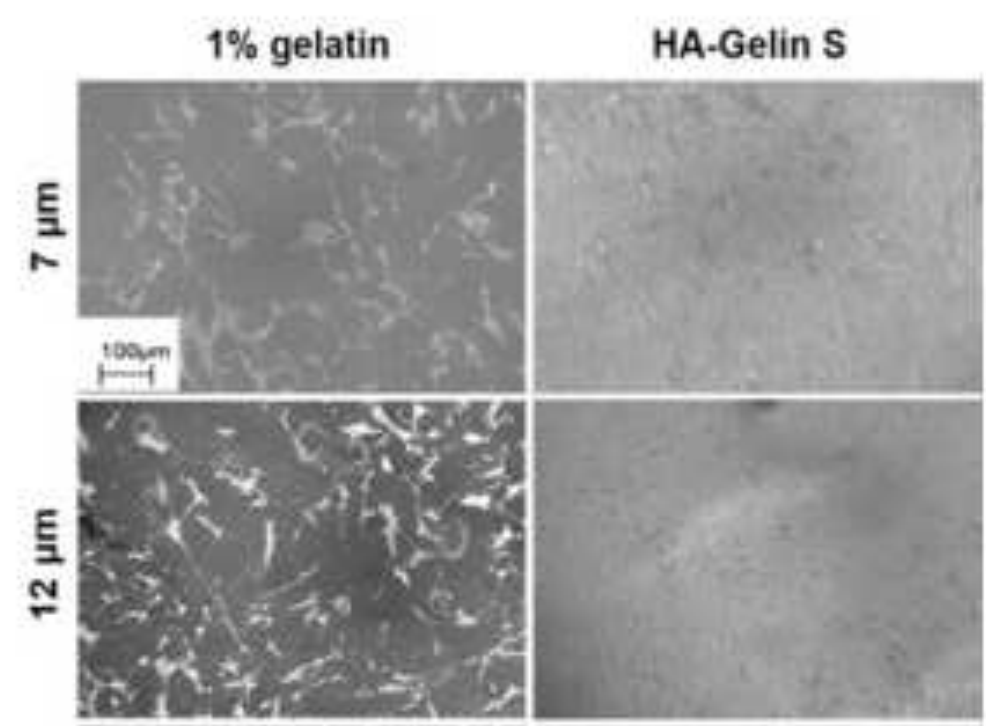

4

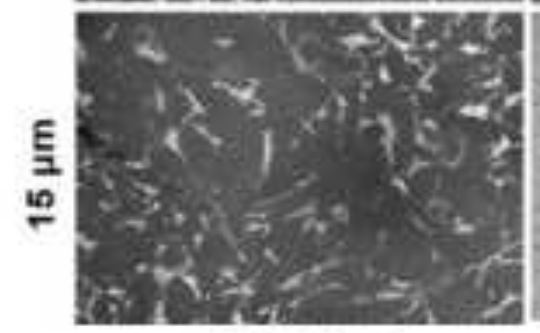

HA-Gelin S

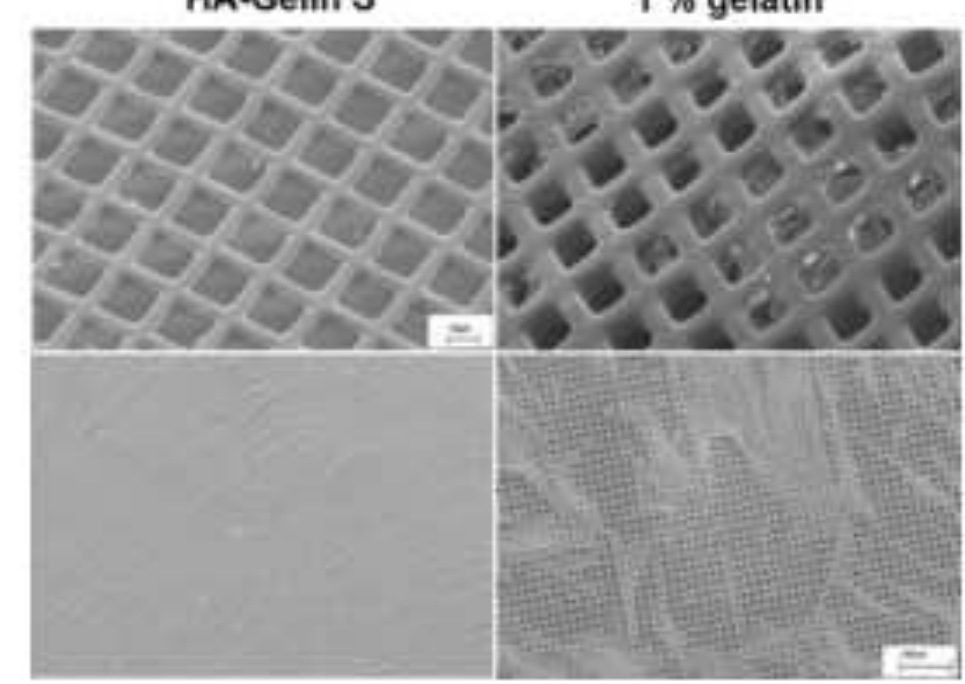

b

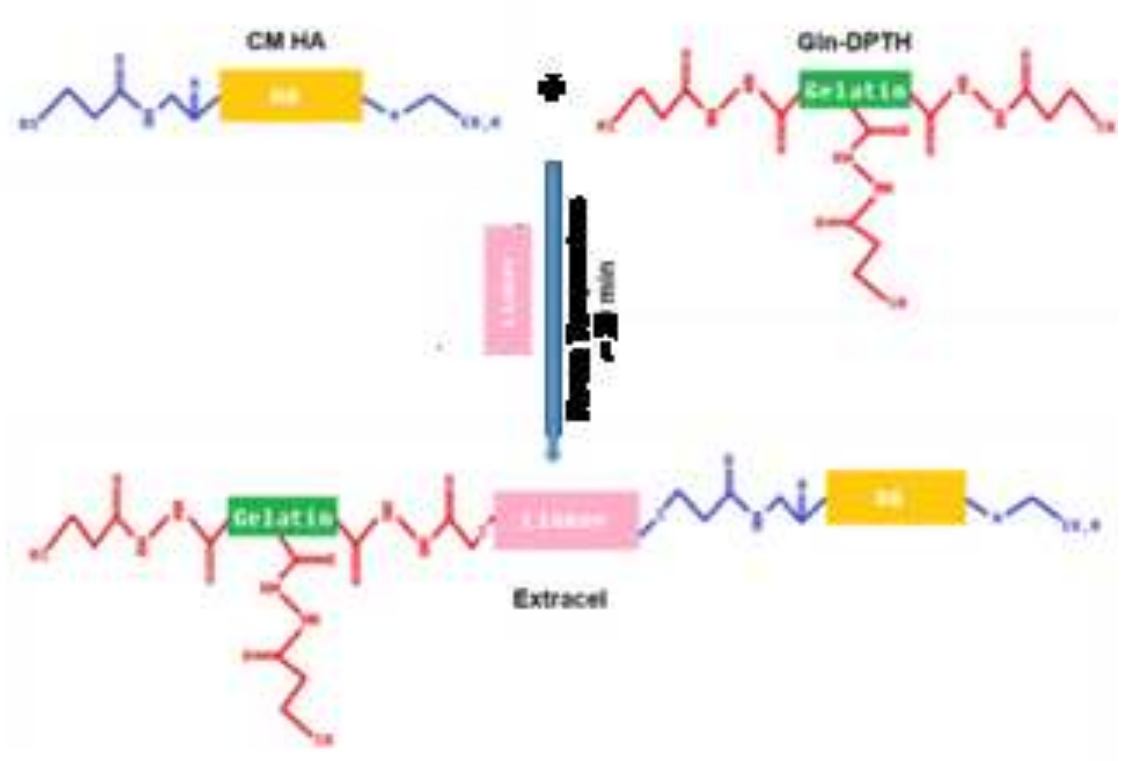

d

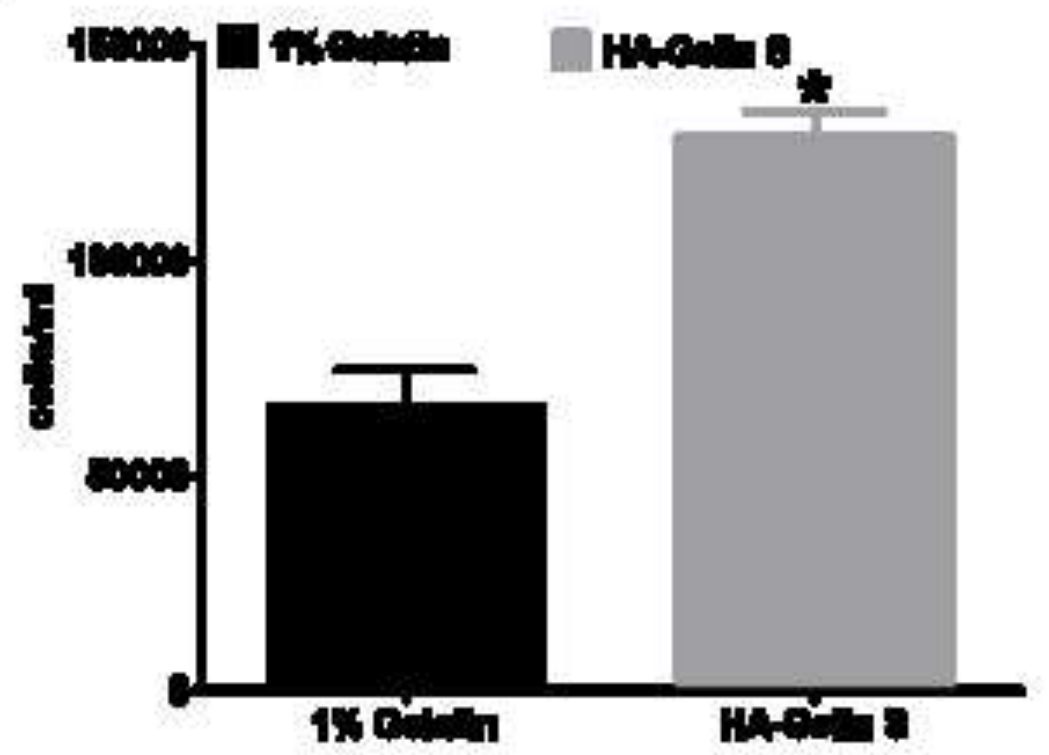


국

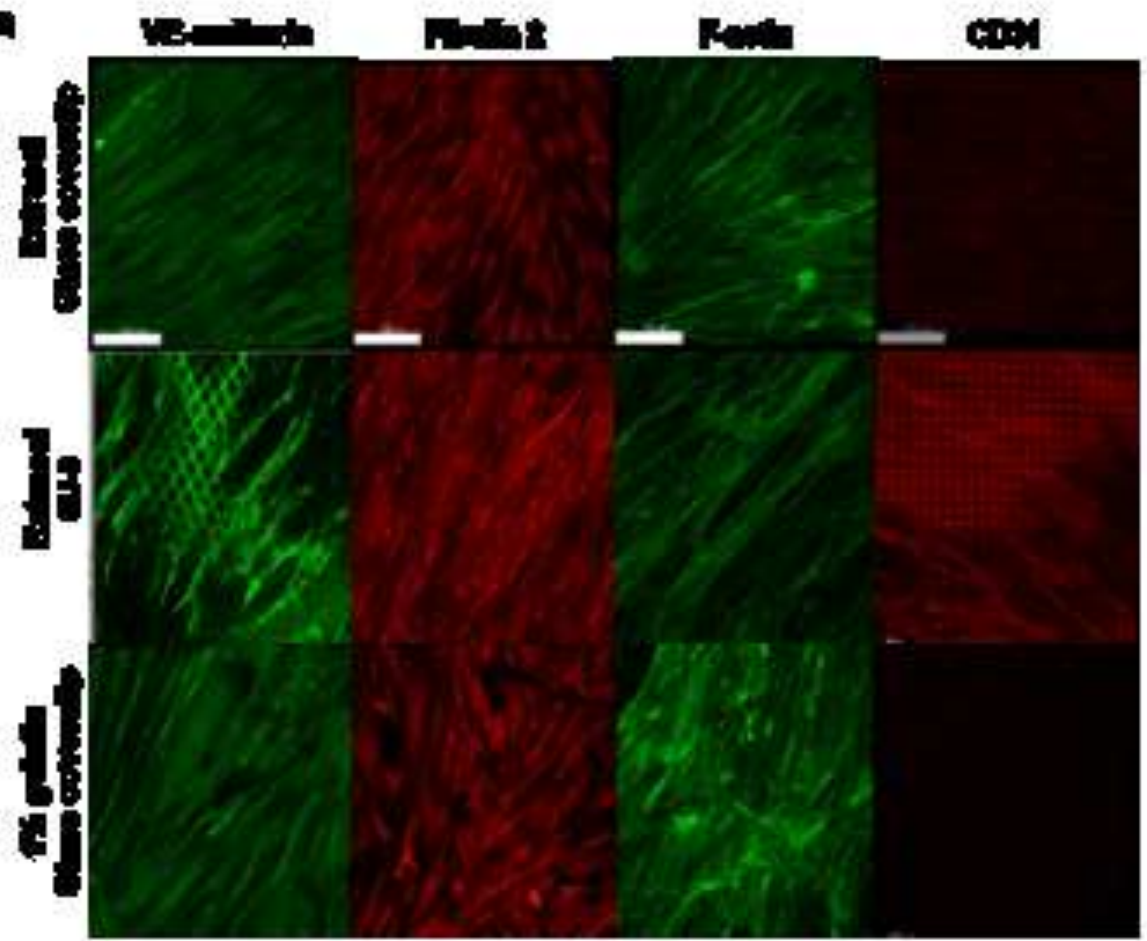

6

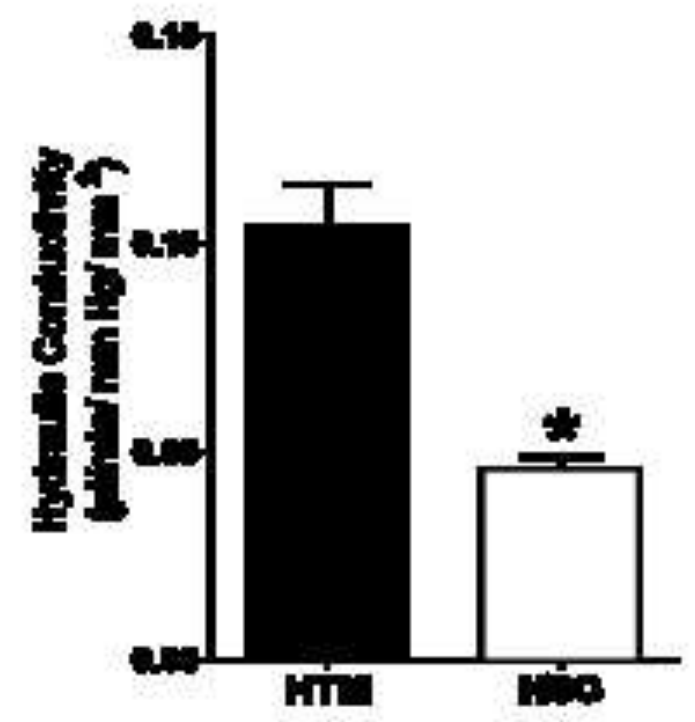

b

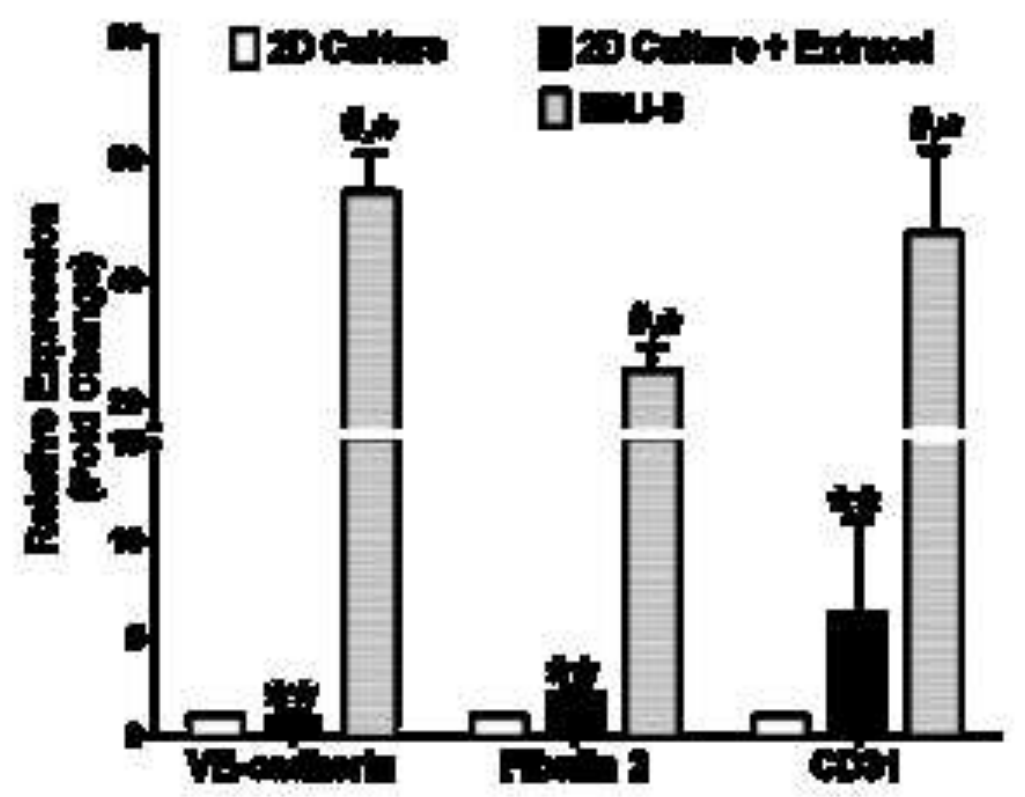

No perfusion

Perfused at $4 \mu \mathrm{l} / \mathrm{min}$

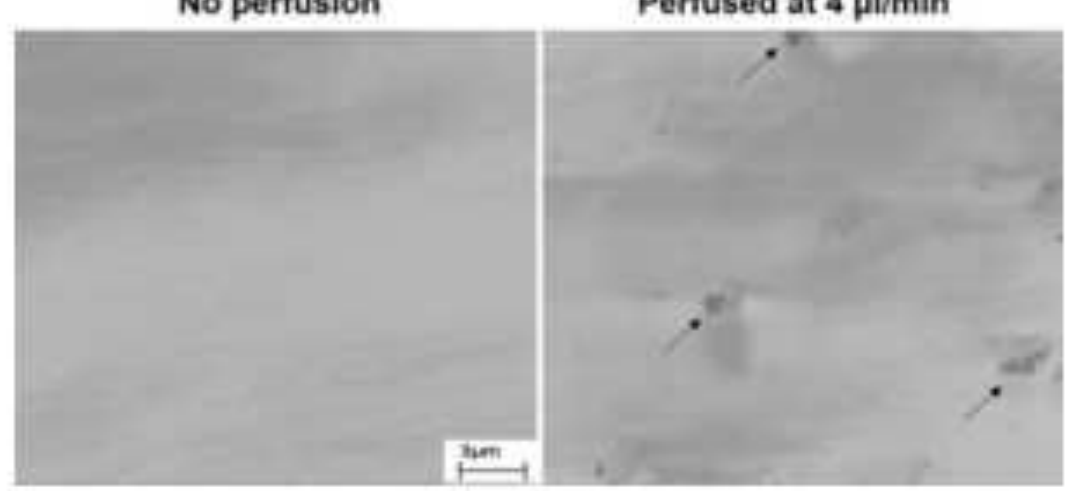


Figure 3

-
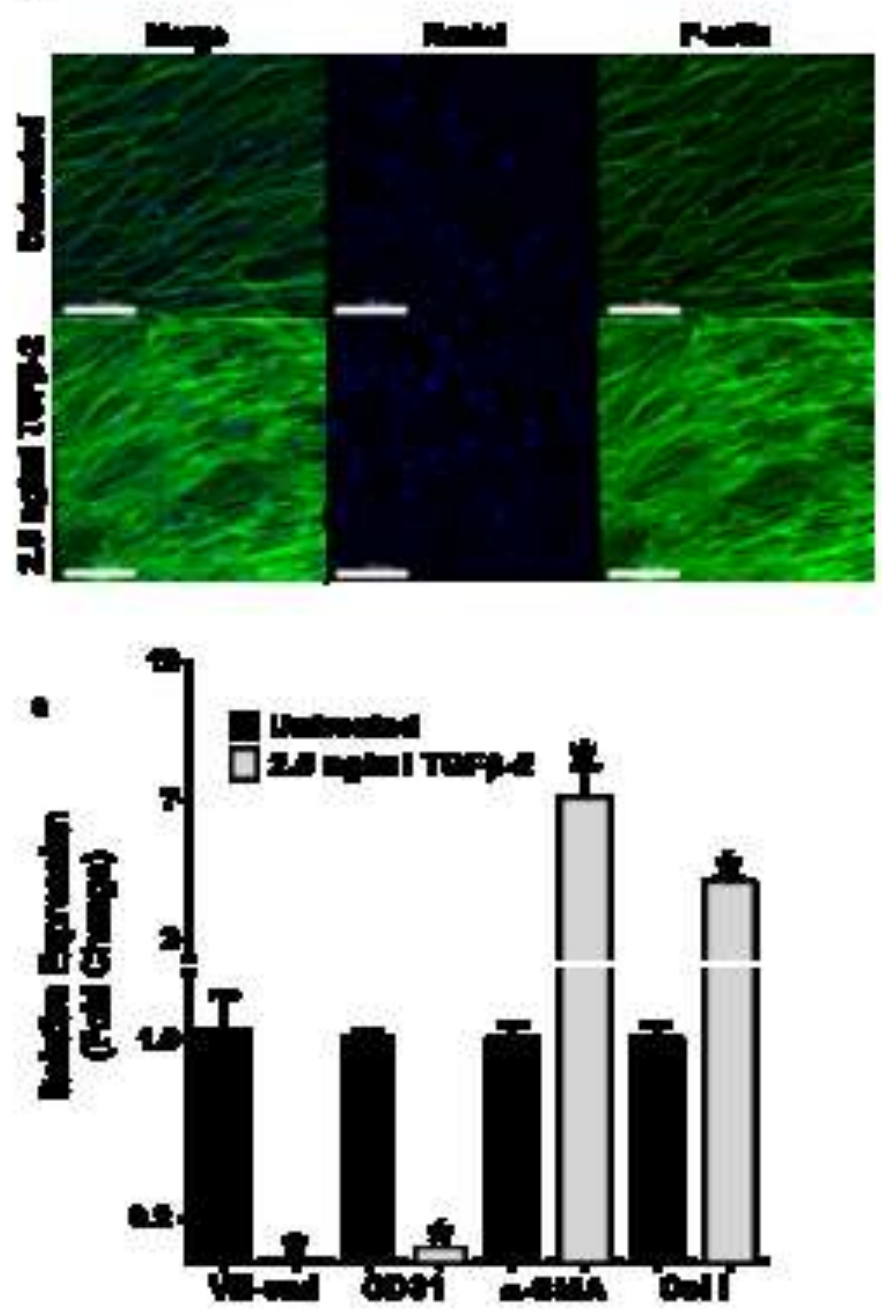

-

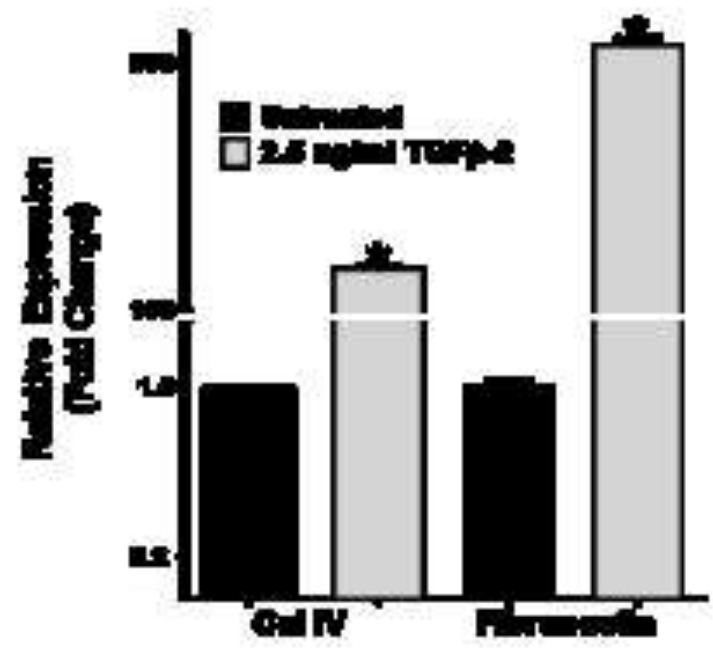

-

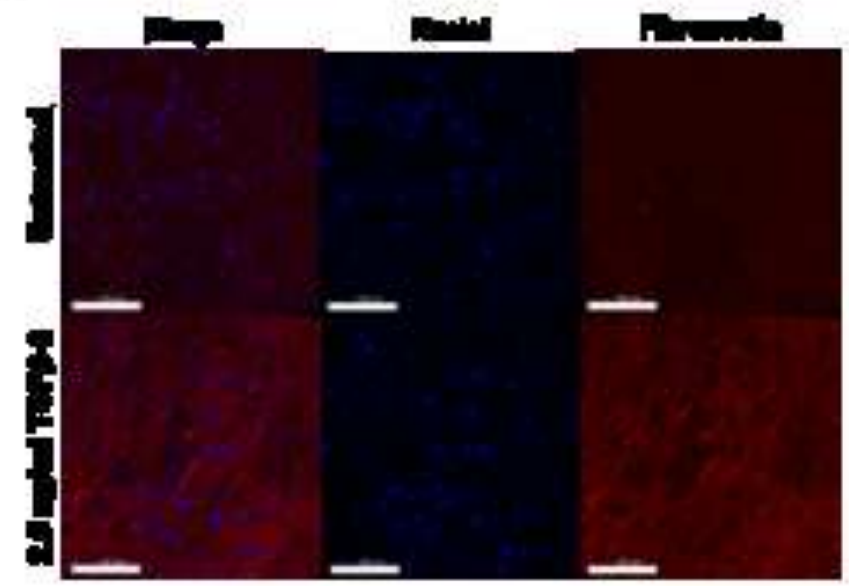

-

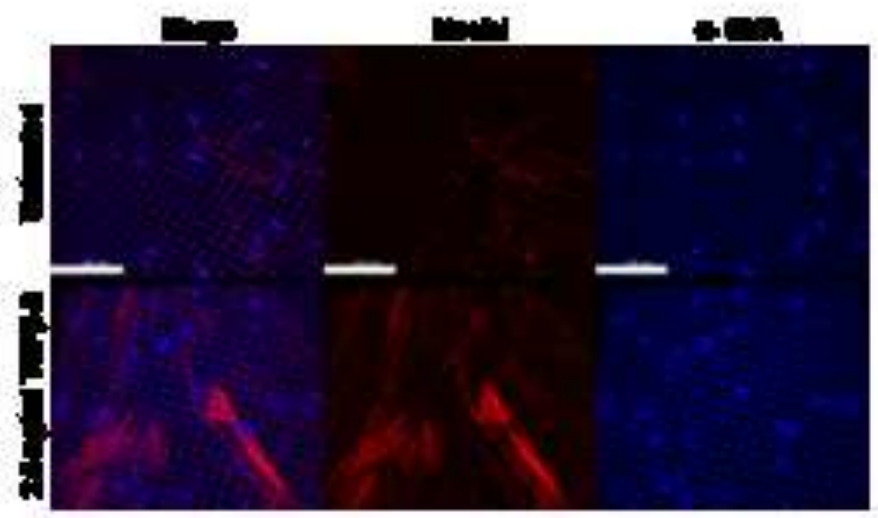

I

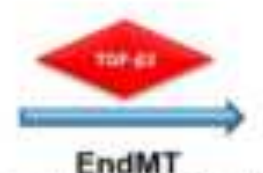

EndMT

Endothelial markers (CD 31, VE-cadherin) ta-SMA

fFibronectin, Collagens (I, IV) 


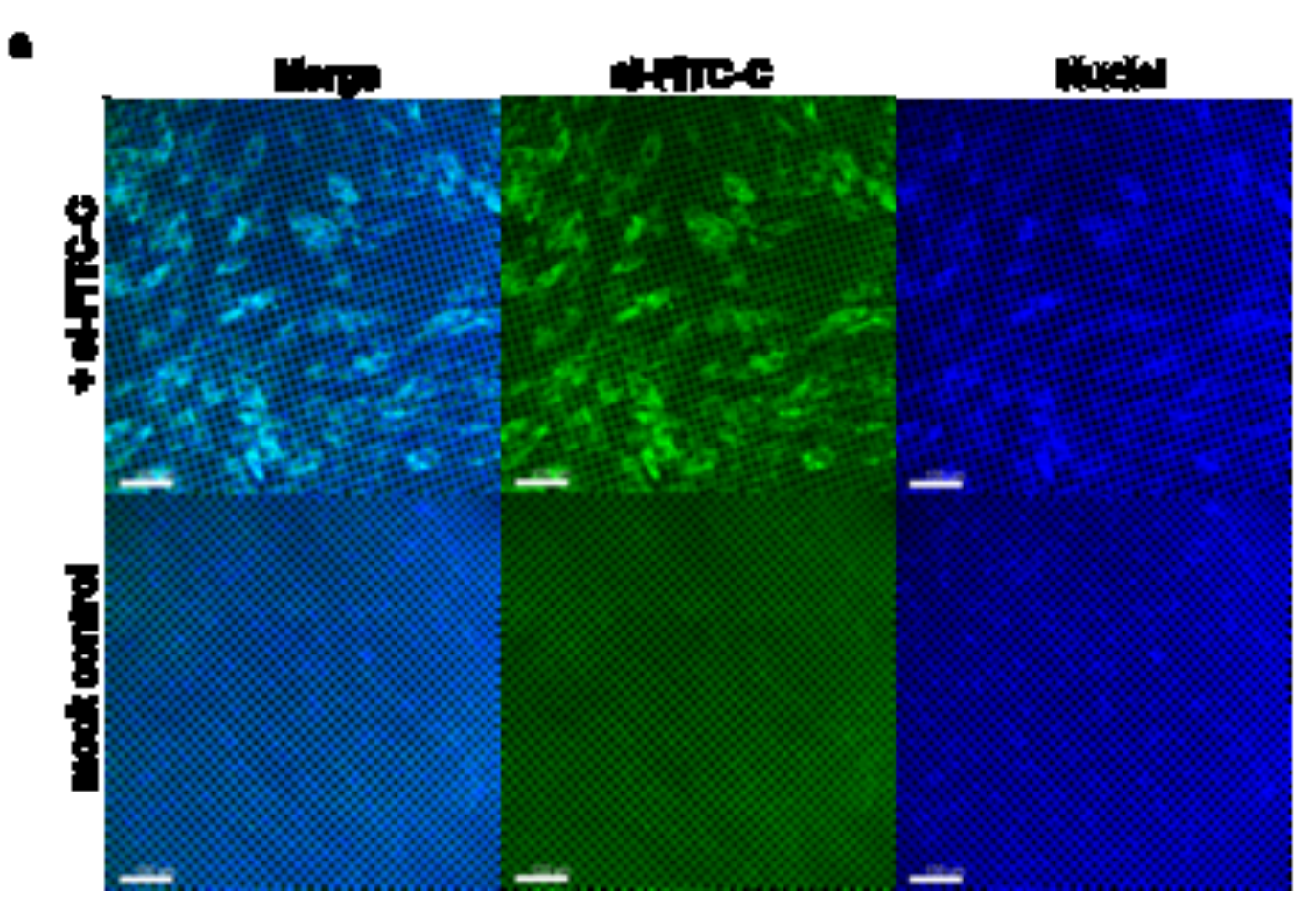

$b$

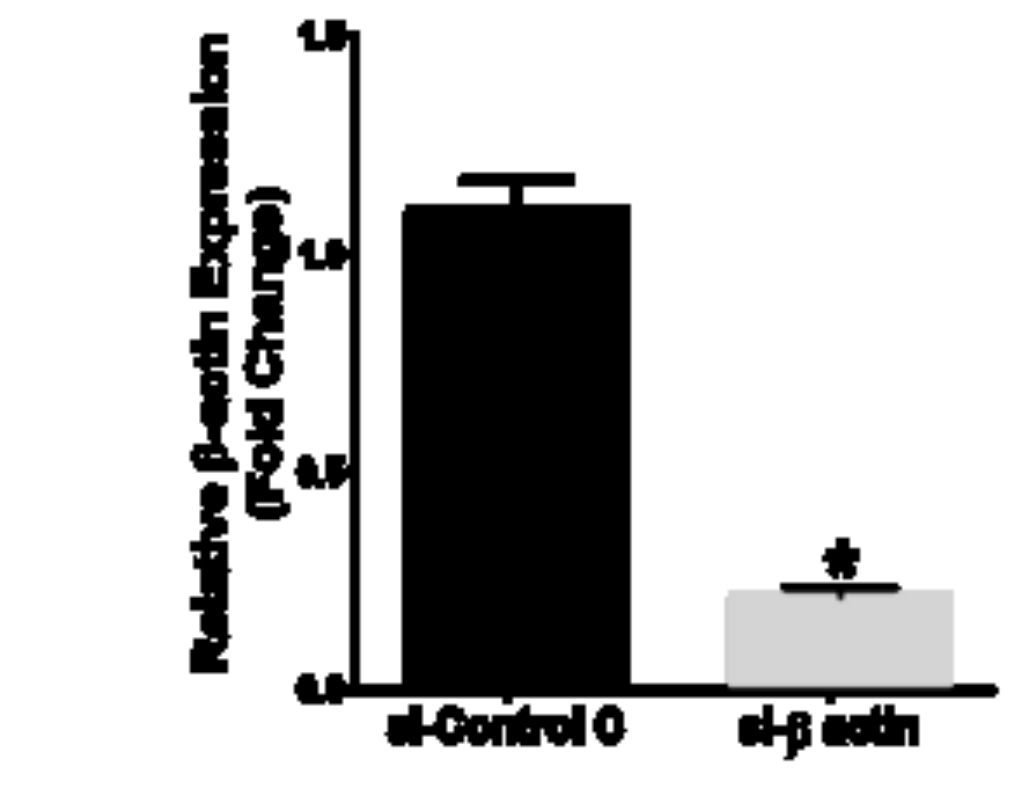

b

,

. 